\title{
Die Bedeutung des Göttweiger Interstadials im Ablauf der Würm-Eiszeit
}

\author{
Von Hugo Gross, Bamberg
}

Zus a mmenfassung. Das im Würm-Vereisungsgebiet in Oberbayern bei Hörmating entdeckte Drumlin-Profil, das E. EBERS (in diesem Jahrbuch) stratigraphisch, paläoklimatologisch und chronologisch ausgewertet hat, läßt sich genau mit dem typischen Löß-Profil im östlichen Niederösterreich, insbesondere mit seinem Fellabrunner (Göttweiger) fossilen Bodenbildungskomplex im Sinne von F. BrandtNer parallelisieren. Die Zeit der Göttweiger Verlehmung ist das Hörmatinger Interstadial von ca. 47000 (oder 48000) vor heute bis zum Abschluß durch eine Torfschicht mit dem C14-Datum Gro-2593: 45300 1000 v. h. Da manche Befunde für mindestens e in e n Kälterückschlag sprechen, ist wohl der Name "Göttweiger Interstadial-Komplex“ passender. Diese Unterbrechung des glazialen Klimas in der Würm-Eiszeit dauerte von etwa 47000 (oder 48000) bis ca. 30000 v. h. Die Vorlandvergletscherung wurde durch die Schüttung eines Vorstoßschotters eingeleitet, auf dem sehr wahrscheinlich im Paudorfer Interstadial die Innerwürm-Verwitterungsschicht (E. KRAUS) entstand. Die Vorlandvergletscherung erfolgte allem Anschein nach von der Aare in der Schweiz bis zur Salzach ganz (oder fast ganz) im Hauptwürm; ihr ging eine mehrfache Schüttung älterer Würmschotter aus den Alpentälern voran. Diese älteren Würmschotter (in Hörmating mit 2-3 m mächtiger Verwitterungsrinde) können 2-3 interstadiale Schieferkohlenflöze enthalten, von denen das älteste an mehreren Orten dem letztinterglazialen Flöz aufliegt.

$\mathrm{S} \mathrm{u} \mathrm{m} \mathrm{m}$ a $\mathrm{r}$. The drumlin section recently discovered near Hörmating in the Upper Bavarian area of the Würm Glaciation and evaluated with regard to stratigraphy, paleoclimatology, and chronology by E. EBers (in this annual) can closely be correlated with the typical loess section of eastern Lower Austria, particularly with its Fellabrunn (Göttweig) fossil soil complex as described and interpreted by F. BrandTNER. The Göttweig loamificaton developed during the Hörmating Interstadial from c. 47000 (or 48000) until the close marked by a thin peat layer $45300 \pm 1000 \mathrm{C}-14$ years old. As some findings are suggestive of at least o $\mathrm{n}$ e cold oscillation, the term "Göttweig Interstadial Complex" appears to be correcter. This period interrupted the glacial climate of the Würm Glaciation from c. 47000 (or 48000 ) until c. 30000 B. P. The northern alpine Piedmont Glaciation was initiated by an advance-gravel later on weathered on the surface probably during the Paudorf Interstadial and advanced entirely (or almost entirely) during the Main Würm Stage from the (Swiss) Aare to the Salzach rivers, after several. Lower Würm gravel masses had poured out of the Alps valleys during the Early Würm Stage. This Lower Würm gravel was capped by a weathered (decalcified) stratum 2-3 metres in thickness and can contain up to three interstadial Schieferkohle (Pleistocene lignite) beds intercalated, the lowermost overlying a Schieferkohle stratum deposited during the Riss-Würm Interglacial at some sites.

Angelpunkt der Gliederung und Chronologie der Letzten Eiszeit auf Grund der Lößstratigraphie ist bekanntlich in den Trockengebieten des südöstlichen Mitteleuropa die noch immer umstrittene mächtige Göttweiger fossile Bodenbildung, nach W. Soergel der Beweis für eine besonders lange interstadiale Unterbrechung des glazialen Klimas, seine $\mathrm{Haupts}$ ch wankung Würm I, Würm I I, im neuesten quartärgeologischen Schrifttum als Göt t w e ige r Inter sta d i a l (F. E. ZEuner 1954, S. 103) allgemein bekannt.

Lange Zeit wurde von den Quartärgeologen als Bildung dieses Interstadials im Lößprofil nur die sogen. "G öt twe ig er Ver lehm ung s z on e " angesehen. 1954 haben aber F. Brandtner und J. Fink im ariden östlichen Niederösterreich die vollständ i g e Ausbildung des Göttweiger fossilen Bodens entdeckt: eine Göttweiger Verlehmungszone, auf der eine dünne (öfters fehlende) Lößschicht und darüber drei mit Lößschichten wechsellagernde Humuszonen liegen (Fellabrunner Bodenbildungskomplex von F. Brandtner $=S$ tillf ried A - K omplex von J. FinK), die schon im etwas humideren westlichen Niederösterreich (z. B. in Göttweig) bis auf einen unbedeutenden Rest oder ganz denudiert sind. Im Gegensatz zu E. SchönhaIs (1956) und tschechischen Forschern haben F. Brandtner (zuletzt 1956) und J. Fink (zuletzt 1956) die 
Annahme eines genetischen Zusammenhangs zwischen der Verlehmungszone und dem darauf liegenden oben genannten Schichtpaket abgelehnt und seine Bildung auf dreimalige Aufwehung von Lößschichten zurïickgeführt, die in humideren Zwischenzeiten jeweils teilweise oder auch ganz in Schwarzerde (Humuszonen) umgewandelt seien.

Die Untersuchung des Fellabrunner Bodenbildungskomplexes in Oberfellabrunn (F. Brandtner 1954, S. 58, Abb. 2, Fig. 1) mit Hilfe der C14-Methode (Hl. de Vries $1959 \mathrm{a}$ und b) ergab für die Schichtenfoslge von der untersten Humuszone bis in den humosen Löß (sicher der dritten, obersten Humuszone entsprechend) im basalen Teil des Jüngeren Löß II die Zeitstellung $41900 \pm 800$ (oder $42700 \pm 1400$ ) bis $31600 \pm 500$ v. h. (vor heute). Die Göttweiger Verlehmungszone, die für die C14-Datierung der Verlehmung nicht genïgend Humus enthielt, wollte Hl. de VRIEs (1959a) offenbar auf Grund des ursprünglichen C14-Datums (Gro-1217: 48300 2000) für eine Holzkohle-Probe u n t e r der Verlehmungszone von W I/II in Senftenberg (Niederösterreich) eher in ein Interstadial um 48000 v. h. als in ein älteres stellen; ein solches Interstadial war aber damals noch nirgends bekannt.

Nun hat E. EBers (1960, in diesem Jahrbuch) 1959 e in Gegenstü ck z u de m Fellabrunner Bodenbildungskomplex des Göttweiger Inter$s$ tadials im Würm-Profil von Hörmating im würmeiszeitlichen InnGlets chergebiet ca. $10 \mathrm{~km}$ innerhalb der innersten (Olkofener) Jung-Endmoräne und ca. $23 \mathrm{~km}$ von der Ausmündung des alpinen Inn-Tales ins Vorland entdeckt. Es handelt sich dabei um die Schichtenfolge von der Oberkante der unteren Verwitterungsschicht bis zur Unterkante der interstadialen Seekreide mit dünner Torfdecke (C14-Alter $45300 \pm 1000$ Jahre). Auf Grund der zur Verfügung stehenden C14-Daten liegt folgende (zeitliche und paläoklimatische) Parallelisierung mit den Schichten des Fellabrunner Bodenbildungskomplexes von Oberfellabrunn nahe:

1. Würm-Basalschotter in Hörmating: unverwitterter Jüngerer Löß I von W $I$ in Oberfellabrunn (und Senftenberg); Klima glazial;

2. Hörmatinger Interstadial (Torf auf ca. $80 \mathrm{~cm}$ Seekreide): Verwitterung der obersten Schicht des Jüngeren Löß I zur Göttweiger Verlehmungszone; Klima kühl-temperiert, feucht (Mittelgebirgs-Klima) von ca. 47000 (oder 48000 ) v. h. bis 45000 v. h.;

3. Schüttung von 2-3 m Würm-Vorstoß-Schotter in Hörmating: Aufwehung der untersten Lößschicht auf der Göttweiger Verlehmungszone; (glazialer) Klimarückschlag von höchstens ca. 45000 bis 43000 v. h.;

4. Erosion des Würm-Schotters 3 auf dem interstadialen Torf, Ablagerung von 2-4 m Seeton auf ihm, Verwitterung dieses Seetons und des Würm-Schotters 3 (physikalische Verwitterung, Entkalkung): Bildung des Schichtpakets aus Humusund Lößschichten auf der Verlehmungszone aus dreimal aufgewehten Lößablagerungen; Klima kühl, interstadial, sehr kontinental mit wechselnder Humidität und abnehmender Temperatur, von ca. 43000 bis ca. 30000 v. h.;

5. Ablagerung des oberen nicht entkalkten Würm-Schotters (Vorstoß-Schotter der Hauptwürm-Vereisung) auf Schicht 4 in Hörmating: Bildung und Ablagerung des Jüngeren Löß II von W II auf dem Fellabrunner Bodenbildungskomplex nach ca. 30000 v. h. in glazialem Klima.

Diese Parallelisierung entspricht genau der von F. Brandtner (1954, S. 66) gegebenen Interpretation der Schichten des Fellabrunner Bodenkomplexes. Da (mindestens) e in (?glazialer) Kälterückschlag die Bildung dieser Schichtenfolge unterbrochen hat, erscheint es zweckmäßiger, von einem Göt tweiger Interstadial-Komplex zu sprechen. Die vorgeschlagene Parallelisierung müßte noch durch weitere Untersuchungen überprüft werden; dafür sind vor allem $\mathrm{C}^{14}$-Messungen von Humuszonen auf der Göttweiger 
Verlehmungszone und von einwandfreien Holzkohle-Proben a uf ihr erwünscht, ferner müßte nach ähnlichen Aufschlüssen (Kiesgruben, besonders in Drumlins) wie in Hörmating gefahndet werden. Das Hörmatinger Interstadial paßt aber, wie es Hl. de VRIES (1959a) postuliert hat, zeitlich und stratigraphisch so gut als Bildungszeit der Göttweiger Verlehmungszone, daß die obige Parallelisierung höchst wahrscheinlich richtig ist. Dann würde der Beginn des Göttweiger Interstadial-Komplexes mit 47000 oder 48000 v. h. und das Ende der Göttweiger Verlehmung (also die Zeitstellung der Oberkante der Verlehmungszone) mit ca. 45000 v. h. anzusetzen sein. Dazu paßt das alte Datum (ca. 48300 v. h.) für die Senftenberger Holzkohle, wenn sie un te $r$ der Verlehmungszone gelegen hat (wie F. BRANDTNER noch heute versichert), aber auch das neue Datum Gro-1771: „älter als 54000 Jahre“ (J. FINK 1959 S. 60) widerlegt, wenn es wirklich einwandfrei ist, die Behauptung von J. FINK (1959), daß sie a u f der Verlehmungszone gelegen habe. ${ }^{1}$ )

Manche Quartägeologen halten auch heute daran fest, daß das Göttweiger Interstadial durch seine Verlehmungszone allein repräsentiert werde und daß das Schichtpaket mit den 3 Humuszonen auf ihr das „Frühglazial FW a-c der Würmeiszeit“ (K. BRuNNACKer 1957, S. 85), d. h. die „beginnende letzte Kaltzeit“ (J. FINK 1956, S. 49) bedeute, während es nach F. Brandtner (1954, S. 66) und P. Woldstedt (1958 a, S. 153, und 1958 b, S. 245) den Ausklang des Göttweiger Interstadials erkennen läßt. Für die Festlegung seines Endes um \pm 30000 v. h. spricht eine ganze Reihe von C14-Daten (P. WolDSTEDt 1958 a, S. 153, H. Gross 1958, S. 167, 167, 171), vor allem die C14-Daten des Karrestobel- Torfs, ferner der von P. BECK in seine Spiezer Schwankung W I/II gestellten Schieferkohle im Glütschtal bei Thun (B-20: $29000 \pm 1500$ v. h. nach H. OEschGER \& Mitarb. 1959, S. 138) und der Olschewien-Kulturschicht (Aurignacien II L. VÉrTes) Gro-1935: $30710 \pm 600$ v. h. (nicht „v. d. Zw.“ d. h. v. Chr., wie L. Vértes 1959 infolge eines Übersetzungsfehlers angegeben hat), denn dieses C14-Datum gilt wenigstens annähernd auch für die Olschewien-Kulturschicht in der Potočka-Bärenhöhle in den Ostkarawanken in $1700 \mathrm{~m}$ Höhe ü. d. M., weswegen diese Begehung der Höhle von A. PENCK (1939) ins Riß/Würm-Interglazial gestellt worden ist. Um $\pm 30000 \mathrm{v}$. h. ist aber in Oberfellabrunn bereits Löß gebildet und abgelagert worden, so daß die Annahme berechtigt ist, daß die Wechsellagerung von Humus- und Lößschichten im zweiten Teil des Göttweiger Interstadial-Komplexes viel mehr auf Schwankungen der Humidität als der Temperatur zurückzuführen und Denudation dieses Schichtpakets auf der Göttweiger Verlehmungszone schon im humideren westlichen Niederösterreich nicht durch Solifluktion. sondern durch Abspülung bewirkt worden ist.

Das C14-Datum $41900 \pm 800$ v. h. ist in Groningen nicht nur für die unterste Humuszone auf der Göttweiger Verlehmungszone in Oberfellabrunn gemessen worden, sondern auch für den beginnenden Rückzug des (jüngeren) Irish Sea Glacier in England nordwestlich von Birmingham (vgl. H. Gross 1959). Das Interstadial (mit Picea!) von Chelford (Cheshire) im Liegenden der Grundmoräne dieses ersten Inlandeises, das nach dem Eem-Interglazial dieses Gebiet erreicht hat, ist etwas mehr als 57000 C14-Jahre alt (F. W. Shotton 1959) und hatte nach den paläobotanischen Befunden (I. M. Simpson \& R. G. West 1958) und nach der Käferfauna (G. R. Coope 1959) ein Klima wie in SüdFinnland, es dürfte, da solche alten Daten als Minimaldaten gelten müssen, dem BrörupLoopstedt-Interstadial zeitlich entsprechen. Nach den C14-Daten 57000 und 41900 v. h. ist der Vorstoß dieses Irish Sea Glacier in das W I-Maximum zu stellen. Sein Rückzug

1) Prof. de Vries hat schon vor der Veröffentlichung seiner C14-Datenliste von 1958 die Angabe von J. FINK gekannt, sie aber ignoriert, weil sonst das ursprüngliche C14-Datum der Senftenberger Holzkohle nicht zu seiner damaligen Datierung 42000 v. h. des Beginns des Göttweiger Interstadials paßte; aus dem gleichen Grunde habe auch ich das getan, da ich das C14-Datum aus der Datenliste von Hl. de Vries (1958) nehmen mußte. Diese Nichtbeachtung ist außerdem gerechtfertigt durch die neue Datierung der Senftenberger Probe und des Beginns des Göttweiger Interstadial-Komplexes. 
erfolgte im zweiten Teil des Göttweiger Interstadial-Komplexes; der nächste Inlandeisvorstoß (Welsh Re-advance), der aber das Gebiet nordwestlich von Birmingham nicht erreicht hat, folgte erst nach mehreren Tausend Jahren im späten Aurignacien (vgl. H. Gross 1959). In der Grundmoräne des Irish Sea Glacier an seinem Südrand bei Penkridge (Staffordshire) ist ein Toteisloch mit einem Moor durch Tieftauen einer Toteismasse zu Beginn des Alleröd-Interstadials, also vor ca. 12000 Jahren, entstanden. Da diese Toteismasse im Anfangsabschnitt des Rückzugs des Irish Sea Glacier, also zu Beginn des zweiten Teils des Göttweiger Interstadial-Komplexes um 42000 v. h., unter Grundmoräne und Schotter verschüttet worden ist, taute dieses Toteis erst nach 30000 Jahren auf (F. W. Sнотton \& I. Strachan 1958, 1959), hat also den zweiten Abschnitt (ca. 43000 bis 30000 v. h.) des Göttweiger Interstadial-Komplexes überdauert ${ }^{2}$ ); dieses kann daher im Optimum höchstens kühl-boreal gewesen sein und nicht interglazial ${ }^{3}$ ). Das gilt zweifellos auch für Mitteleuropa, denn auch im Letzten Interglazial war nach den paläofloristischen und paläofaunistischen Befunden in den sehr fossilreichen fluviatilen EemAblagerungen in London unter dem Trafalgar Square (J. W. FRANKs \& Mitarb. 1958) das Klima in England nicht weniger warm als in Mitteleuropa, nach F. W. SноттоN (1959) nämlich wie heute in Südeuropa. Die C ${ }^{14}-D a t e n$ von Chelford und Upton Warren in England und von Hörmating und Großweil in Oberbayern ermöglichen eine Parallelisierung von Phasen der Weichsel- und Würm-Vereisung wie auch die Alleröd-Bildungen.

Aus Nordwest-England ist bisher noch kein Aquivalent des Hörmatinger Interstadials bekannt. Die Bildung derartiger Ablagerungen ist offenbar durch die stark abkühlende Wirkung des nahen Inlandeisrandes (Irish Sea Glacier) verhindert worden; dazu kommt noch, daß Bewegungen eines Inlandeisrandes einer Änderung des Allgemeinklimas erheblich nachhinken. Im Fall Hörmating war die abkühlende Wirkung des alpinen Eisstromnetzes unvergleichlich viel geringer und wurde sicher noch durch die Föhnwirkung abgeschwächt.

In Holland muß das lange Interstadial W I/II auch das glaziale Klima (mit Decksand-Bildung und -Ablagerung, Solifluktion und Kryoturbation, die dort von Th. van der HAMmen 1951, S. 122-125, sogar noch für die Jüngere Tundrenzeit nachgewiesen ist) unterbrochen haben (vgl. H. Gross 1958, S. 157, 167-170). Aus dem Senkungsgebiet in Holland liegen $\mathrm{C}^{14}$-Daten von Torfschichten mit anscheinend spärlichem Picea-Holz ${ }^{3 a}$ ) aus der Zeit zwischen \pm 40000 und \pm 30000 v. h. vor (S. T. ANDERSEN \& Mitarb. 1960), die mit Rücksicht auf die sehr hohen NBP-Werte für Bildungen einer arktischen pleniglazialen Tundrenzeit gehalten worden sind; die hohen NBP-Werte können aber auch von der Moorvegetation stammen. Der oberste Torf von Breda (Gro-2008: $29930 \pm 300$ und Gro-2007: $31750 \pm 400$ v.h.) ist nach S. T. Andersen \& Mitarb. (1960) in einer kühl-temperierten Waldzeit gebildet und stammt nach den C14-Daten aus dem Ende des Göttweiger Interstadial-Komplexes (das Paudorfer Interstadial ist ca. 3000 Jahre jünger); die C14-Daten Gro-1718: $46500 \pm 2000$ und Gro-1715: $44500 \pm 1500$ v. h. für pleniglaziale Torfschichten, die einer arktischen Tundrenzeit zugeschrieben wurden, stammen aus der Zeit des Hörmatinger Interstadials.

2) Für die Zusendung eines Sonderdruckes dieser und anderer einschlägiger Arbeiten aus seinem Geolog. Institut der Universität Birmingham bin ich Herrn Prof. F. W. SнOтton zu großem Dank verpflichtet.

3) Auf dem Festland begann bei Plön in Ho'stein verschüttetes Toteis (wahrscheinlich aus dem Pommerschen Stadium) erst am Ende der frühen Wärmezeit (um 5500 v. Chr.) aufzutauen (K. Gripp \& R. SchütrumpF 1953).

3a) Nach briefl. Mitteil. vom 18. 7.60 von Herrn Dr. W. H. ZaGwiJn in Haarlem handelt es sich aber um geringmächtige \pm sandig-tonige humose Süßwasser-Sedimente, in die bisweilen Holz

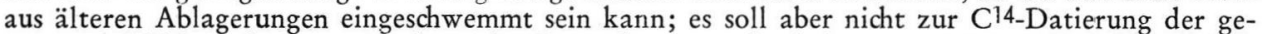
nannten Schichten benutzt worden sein. Dann könnten die hohen NBP.-Werte wohl von einer üppigen Sumpf-Vegetation hẹrstammen. 
Jie lakustrischen Ablagerungen aus dem Göttweiger Interstadial-Komplex in Hör.ng scheinen für die Existenz eines interstadialen Rosenheimer Sees oder Seengebiets prechen. Ebenso ist der Karrestobel-Torf nördlich von Ravensburg allem Anschein die Uferbildung eines Sees aus dem gleichen Interstadial im Schussental. In beiden ieten sind aber noch weitere Untersuchungen nach dieser Richtung notwendig; insbelere müßte im Karrestobel noch geprüft werden, ob das Liegende seines Torfes wirkWürm- (W I-) und nicht Altmoräne ist.

Jas Hörmatinger Interstadial ist nach E. EBERs (1960) auch deswegen interessant, das gepreßte Picea-Holz im Torf älter als dieser (nämlich älter als 53000 C 14-Jahre) die Pollenspektra beweisen aber, daß Picea im Hörmatinger Interstadial als Wald$\mathrm{n}$ vorkam). Dieses Holz muß daher aus einem der verbreiteten interstadialen oder :glazialen Schieferkohlen-Flöze des würmeiszeitlichen Inn-Gletschergebiets in das sstadiale Hörmatinger Wasserbecken gespült sein. Das Gleiche ist anscheinend auch ranchen an Kleinholz auffallend reichen Wasserburger Schieferkohlen-Flözen (Stadt iserburg, Zell am Inn, Inn-Steilufer zwischen Königswart und Bergholz südlich von s) der Fall ${ }^{4}$ ).

_eider ist das Liegende des Hörmatinger Würm-Basalschotters nicht bekannt. Es ist nach dem Vorkommen von älterem eingeschwemmtem Picea-Holz im Hörmatinger rstadial zweifellos statthaft, als Entsprechung der Fortsetzung des Hörmatinger emprofils die Schichtenfolge in dem ca. $65 \mathrm{~km}$ südwestlich entfernten Großwcil bei hel anzunehmen. Hier liegt unter dem Würm-Basalschotter im Liegenden von Hauptm-Moränenbildungen zunächst der, wie HELGA REICH (1953) auf Grund ihrer Poliagramme richtig vermutet hat, frühwürmeiszeicliche Anteil des Schieferkohlen-Flözes Toneinschwemmungen (Pollenzone 10: erstes W I-Stadial, Zone 11: erstes W I-Interial mit 59000 C 14-Jahre alter Holzprobe, Zone 12: zweites W I-Stadial, Zone 13: ites W I-Interstadial, oberste Schicht älter als 50000 C14-Jahre nach Groninger Mes;en 1958 und 1959). Die unteren Schieferkohlenschichten (Pollenzonen 5-9) auf Seeton Resten von Elephas antiquus sind im R/W-Interglazial gebildet. Das etwa $200 \mathrm{~m}$ höliegende Schieferkohlenflöz von Pfefferbichl im Lechgletscher-Stammtrichter in $820 \mathrm{~m}$ ie ü. d. M. begann nach der pollenanalytischen Untersuchung von H. REICH (1953) a Ablagerung erst spät im R/W-Interglazial (Pollenzone $7 \mathrm{~b}$ ) und reicht wohl nur mit Pollenzonen 10-12 in W I hinein; es wird von Ton überlagert, der nach oben in Kiese Sande übergeht (weiter ist über das Hangende des Flözes nichts angegeben). In den endiagrammen von Pfefferbichl und Ohlstadt stellen die Pollenzonen 8 (Picea-Abiesmit Abies-Maximum) und 9 (Picea-Zeit, die in Großweil „fehlt") den Schlußabitt des R/W-Interglazials dar. Die jüngeren Schieferkohlen-Schichten der Pollen2n 10-13 (mit wechselnden Dominanzen von Pinus und Picea und sehr niedrigen Wervon Betula sind im frühen WI gebildet, da Zone 11 in Großweil (mindestens) 20 C14-Jahre alt ist und nach H. REICH (1953) die pollenanalytischen Befunde für Herabdrückung der Vegetationsstufen um 600 bis $1000 \mathrm{~m}$ und mehr sprechen; die adischen Spuren von Alnus, Abies, EMW und Corylus sind sicher mit Ton aus den rglazialen Schichten eingeschwemmt. Alle Schieferkohlen der Pollenzonen 10-13 in 1stens $600 \mathrm{~m}$ ü. d. M. im nördlichen Vorland der Schweizer Alpen dürften ebenfalls ;rühe W I zu stellen, d. h. jünger als das Ende des R/W-Interglazials (ca. 70000 oder 30 v. h.) sein, was durch eine $\mathrm{C}^{14}$-Messung nach Isotopen-Anreicherung nachzuprüfen e (A. HARING \& Mitarb. 1958). Ein Schweizer Analogon zu Großweil und Pfeffer1 ist das Schieferkohlen-Vorkommen von Utznach-Kaltbrunn (W. LüDr 1953, S. 82 f., WOLDSTEDT 1958 b, S. 184, 185, R. HantKe 1959, S. 9-11), ein Schweizer Analogon erhalb der Würm-Vereisung ist beispielsweise das Vorkommen bei Gondiswil-Hüswil

) Ein Analogon dazu ist sicher die im Karrestobel-Gebiet von K. BERTsCH 1925 untersuchte thtenfolge mit letztinterglazialen pflanzlichen Großresten. 
(W. Lüdi 1953, Abb. 13, P. Woldstedt 1958 b, S. 185, 186, Abb. 67): der untere Schieferkohlen-Komplex ist letztinterglazial, der obere W I-interstadial, zwischen beiden liegt eine $8 \mathrm{~m}$ mächtige sandige oder sandig-kiesige 'Zwischenschicht.

Nun hat A. Penck (1909, S. 338, 339) im Liegenden des Großweiler SchieferkohlenFlözes, das er damals in die Achenschwankung gestellt hat, stellenweise Moränen gefunden, die er für ältere Würm-Moräne gehalten hat (weswegen er das Flöz als interstadial auffaßte; bei dem damaligen Stand der Quartärbotanik war eine sichere Unterscheidung von interglazialen und interstadialen fossilführenden Ablagerungen noch nicht möglich). Diese Moränen müssen aber, da der untere Teil des Flözes im R/W-Interglazial gebildet ist (H. REICH 1953), A 1 t moränen sein. Solche unterteufen stellenweise auch die unteren Würmschotter mit den Wasserburger Schieferkohlen am Inn (A. Penck 1909, S. 130-132, 138, 139, besonders Abb. 23), die auf einem Areal von über $7 \mathrm{~km}^{2}$ meist auf tertiärem Flinz liegen und deren untere, nach den paläobotanischen Befunden (J. Schuster 1909) letztinterglaziale Schichten seit der Anhebung des Inn-Wasserspiegels durch den Bau von Staustufen unterhalb von Wasserburg leider nicht mehr zugänglich sind. Auch die Moränen unter den Jung-Endmoränen von Gars am Inn, ferner die schlammigen Grundmoränen im Liegenden des unteren Würmschotters am Inn bei Rosenheim, die A. PENCK (1. c.) für ältere Würm-Moränen gehalten hat, sind sicher Altmoränen, denn weder im Würm-Profil von Hörmating, noch im Aufschluß (mit interstadialem Schieferkohlenflöz) in Breinetsried bei Penzberg (unweit Großweil), noch in Großweil (und Pfefferbichl) ist eine W I-Grundmoräne vorhanden, auch nicht im W-I-Maximum! Daher ist es auch sehr wenig wahrscheinlich, daß die von A. PENCK $(1909$, S. 156, 157) im Liegenden des Laufen-Schotters bei Laufen an der Salzach angegebenen Moränen Würm-Moränen sind. Wenn Nachprüfungen ergeben würden, daß es sich auch dort um Altmoränen handelt, wäre die Begründung für die interstadiale Natur des Laufen-Schotters im Einklang mit den paläontologischen Befunden hinfällig, und der Laufen-Schotter müßte als Vorstoßschotter der Hauptwürm-Vereisung aufgefaßt werden wie der obere Würmschotter (auf der unteren Verwitterungsschicht) im Hörmatinger Würm-Profil.

In Hörmating ist das ganze Schichtpaket auf dieser Verwitterungsschicht aus W I/II im Hauptwürm gebildet, die obere Verwitterungsschicht, die Innerwürm-Verwitterungschicht von E. Kraus (1955), höchst wahrscheinlich im Paudorfer Interstadial, wie auch E. EBers (1960) vermutet hal. Für das ganze bayerische Alpenvorland vom Lech bis zur Salzach darf man annehmen, daß die Vorlandvergletscherung mit den drei Jung-Endmoränen erst im Hauptwürm erfolgt ist, daß also auch im W I-Maximum die Gletscher des Eisstromnetzes noch nicht (oder kaum?) aus den Alpentälern herausgetreten waren. Schon viel früher (1882 bzw. 1986) haben A. PenCK \& E. Brückner (1909, S. 1166 mit Fußnote) "die große Masse der unter den Jungmoränen befindlichen Schotter als ,Untere Glacialschotter', entstanden beim Herannahen der letzten Vergletscherung “, gedeutet, haben aber diese Auffassung zu gunsten der Annahme einer interstadialen Ablagerung aufgegeben, als sie im Liegenden dieser Schotter stellenweise Grundmoränen fanden, die sie (aus oben genannten Gründen höchst wahrscheinlich zu Unrecht) für ältere Würm-Moränen hielten. Zu beachten ist in diesem Zusammenhang die durch das beträchtliche Gefälle bedingte große Reichweite der Schotterschüttung; sie betrug z. B. im Hauptwürm-Maximum im Isar-Tal (von Baierbrunn bis fast nach Freising) nach der Geologischen Karte von Bayern (herausgegeben vom Bayer. Geolog. Landesamt 1954) $45 \mathrm{~km}$ !

Wie in Bayern enthalten auch im nördlichen Vorland der Schweizer Alpen die Unteren Glazialschotter" Schieferkohlen-Flöze, die nach R. HantKe (1959) im Frühwürm abgelagert sind (aber bisweilen noch in die Vorstoßphase von Hauptwürm hineinreichen). Im fast $80 \mathrm{~m}$ hohen Kanderdurchstich-Aufschluß bei Thun (R. HantKe 1959, S. 33-35) liegen auf Riß-Moräne mächtige sandige und kiesige verfestigte Deltaschotter mit großen geschrammten Blöcken (wohl aus dem Spät-Riß) und im Hangenden fluvioglaziale Schot- 
ter und glaziolakustrische Seetonlagen und interstadiale Schieferkohlen (W. LüDr 1953, S. 42, Abb. 8 und S. 40, Abb. 7). Das untere Flöz (Gundelsey) stammt nach dem C14Datum B-20: $29000 \pm 1500$ v. h. (Ofschger \& Mitarb. 1959) aus dem Ende des Göttweiger Interstadial-Komplexes, das obere (Wässerifluh) nach dem C14-Datum c. 27000 (R. Hantke 1959, S. 35) aus dem Paudorfer Interstadial (bei Berücksichtigung des Streumaßes)? Die "Guntelsei-Moräne“ von P. BECK im Liegenden der Gundelsey-Schieferkohle ist nach R. HANTKE (1959, S. 36) sehr wahrscheinlich gar keine (W I-) Grundmoräne. Auch die Vorlandvergletscherung der Schweizer Alpen im NW dürfte erst im Hauptwürm erfolgt sein.

Dann muß auch im W I-Maximum das Eisstromnetz ganz erheblich schwächer entwickelt gewesen sein als im Hauptwürm, und seine Ablation im Göttweiger InterstadialKomplex hat wenigstens in den Randgebieten der Alpen und ihren hoch gelegenen Höhlen die Begehung durch Höhlenbären und Menschen ermöglicht.

Nachdem es gelungen ist, die im spätpleistozänen Lößprofil registrierten Hauptphasen der letzten Vereisung mit den würmeiszeitlichen Schichten von Hörmating im InnGletschergebiet zu parallelisieren, ist nichts dagegen einzuwenden, die Abkürzungen W I-III (aber ohne ihre Konnektierung mit Moränen und den drei letzten Kältezacken der Sonnenstrahlungskurve von Milankovitch durch Soergel) zu verwenden, um (mit der nötigen Vorsicht) ältere Publikationen leichter auswerten zu können.

Es werden seit 1957 von verschiedenen Seiten Versuche unternommen, das Göttweiger Interstadial W I/II älter zu machen, vielleicht in der stillen Hoffnung, es zeitlich doch noch mit dem Riß/Würm-Interglazial zur Deckung zu bringen; man hat mit Hl. DE VRIES besonders an das zweite Würm-Interstadial gedacht, für dessen Ende erst jetzt das $\mathrm{C}^{14}$ Datum ca. 59000 v.h. bekannt gegeben worden ist (S. T. ANDERSEN \& Mitarb. 1960). Wenn man dieses Interstadial mit dem Göttweiger identifizieren wollte, würden sich aber folgende unüberwindbare Schwierigkeiten ergeben:

1. Der Beginn der Bildung und Ablagerung des Jüngeren Lösses I (mit einer stellenweise in der Tschechoslowakei, in Niederösterreich, am Kaiserstuhl in Baden und in NordFrankreich gefundenen, höchst wahrscheinlich interstadialen Lehmschicht) würde dann an den Anfang der Würm-Eiszeit herangerückt; das Klima vom späten Riß/Würm-Interglazial bis ziemlich weit in Altwürm hinein war aber, wie allgemein anerkannt wird, sehr humide und daher "lößf eindlich“.

2. Die Oberkante dieser vermeintlichen Göttweiger Verlehmungszone hätte dann ungefähr die Zeitstellung 59000 v.h., während die unterste Humuszone recht dicht über jhr in Ober-Fellabrunn die Zeitstellung ca. 43000 v.h. hat. Da ein solcher Zeitabstand aber ausgeschlossen erscheint, müßte man annehmen, daß alle Ober-Fellabrunner C ${ }^{14}$-Daten durch jüngere organische Substanzen um ca. 16000 Jahre verjüngt sind. Dann müßte man aber auch alle bisher gemessenen zahlreichen jungpaläolithischen $C^{14}$-Daten aus Höhlen- und Lößprofilen aus der Zeit vor mehr als 25000 Jahren für viele Tausend Jahre zu

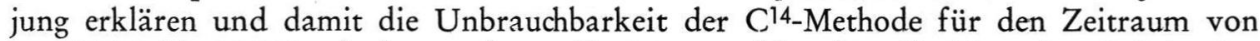
ca. 25000 bis 70000 v.h. konstatieren. Dagegen spricht aber die Tatsache, daß in den allermeisten Fällen einwandfreie Proben, die aus stratigraphischen oder archaeologischen Gründen wenigstens annähernd gleich alt sind, auch ein annähernd gleiches $\mathrm{C}^{14}$-Alter haben; ich verweise auf die $\mathrm{C}^{14}$-Chronologie des Jungpaläolithikums in West- und Mitteleuropa von H. L. Movius, Jr., in "Current Anthropology“ 1, H. 5/6, 1960 (Chicago).

Zur Kontrolle müßten endlich aber mehr Proben aus der untersten Humuszone verschiedener Lößprofile auf der Göttweiger Verlehmungszone mit der $\mathrm{C}^{14}$-Methode datiert werden. Es ist sehr zu bedauern, daß 1958-1959 in Groningen ca. 1000 andere Proben datiert worden sind und daher für die Messung würmeiszeitlicher Proben aus der Zeit zwischen $25000-70000$ v.h. so wenig Zeit zur Verfügung gestanden hat. 


\section{S chriftenverzeichnis:}

Abkürzungen: $\mathrm{EuG}=$ Eiszeitalter und Gegenwart (OOhringen)

Andersen, S. T., Vries, Hl. de, Zagwijn, W. H. (1960):Climatic change and radiocarbon dating in the Weichselian Glacial of Denmark and the Netherlands. - - Geol. en Mijnbouw 39, 38-42.

BRANDTNER, F. (1954): Jungpleistozäner Löß und fossile Böden in Niederösterreich. - - EuG 4/5, 49-82. - - (1956): Lößstratigraphie und paläolithische Kulturabfolge in Niederösterreich und in den angrenzenden Gebieten. - - EuG 7, 127-175.

Brunnacker, K. (1957): Die Geschichte der Böden im jüngeren Pleistozän in Bayern. - Geolog. Bavarica 34, München.

Coope, G. R. (1959): A Late Pleistocene insect fauna from Chelford, Cheshire. - Proceed. R. Soc., $\mathrm{B}, 151,70-86$.

Ebers, Edith (1955): Hauptwürm, Spätwürm, Frühwürm und die Frage der älteren Würmschotter. - EuG 6, 96-109. - - (1960): Drumlinkerne, ältere Würmschotter und das WürmInterstadialprofil von Hörmating in Oberbayern. - EuG 11, 64-76.

FINK, J. (1956): Zur Korrelation der Terrassen und Löße in Ósterreich. - EuG 7, 49-77. - - (1959): in F. Felgenhauer, J. Fink, H1. de Vries 1959, 39-69.

Felgenhauer, F., Fink, J., de Vries, Hl.: Studien zur absoluten und relativen Chronologie der fossilen Böden in Ớsterreich. I Oberfellabrunn. - Archaeol. Austriaca 25, 35-73.

Franks, J. W., Sutcliffe, A. J., Kerney, M. P., Coope, G. R. (1958): Haunt of elephant and rhinoceros: the Trafalgar Square of 100000 years ago - new discoveries. - The Illustr. London News, June 14, 1011-1013.

Gross, H. (1958): Die bisherigen Ergebnisse von C14-Messungen und paläontologischen Untersuchungen für die Gliederung und Chronologie des Jungpleistozäns in Mitteleuropa und den Nachbargebieten. - EuG 9, 155-187. - - (1959): Noch einmal: Riß oder Würm? - EuG 10, 65-76.

Hammen, Th. van der (1951): Late-glacial flora and periglacial phenomena in the Netherlands. Leidse Geolog. Mededel., deel 17, 71-183.

HantKe, RenÉ (1958): Die Gletscherstände des Reuß- und Linthsystems zur ausgehenden Würmeiszeit. - Eclogae Geol. Helvet. 51, 119-149. - - Zur Altersfrage der Mittelterrassenschotter - die riß/würm-interglazialen Bildungen im Linth/Rhein-System und ihre Aquivalente im Aare/Rhone-System. - Vierteljahrsschr. d. naturf. Ges. Zürich 104, 2-47.

Haring, A., de Vries, A. E., de Vries, Hl. (1958): Radiocarbon dating up to 70000 years by isotopic enrichment. - Science 128, 472-473.

Kraus, E. (1955): Zur Zweigliederung der südbayerischen Würmeiszeit durch eine InnerwürmVerwitterungsperiode. - EuG 6, 75-95.

LüDI, W. (1953): Die Pflanzenwelt des Eiszeitalters im nördlichen Vorland der Schweizer Alpen. Veröff. Geobotan. Institut Rübel in Zürich 27, 5-208, Bern.

Oeschger, H., Schwarz, U., \& Gfeller, Chr. (1959): Bern radiocarbon dates I. - Amer. J. of Science Radiocarbon Supplement 1, 133-143.

Penck, A. (1909) in: A. Penck \& E. BrüCKNER (1909). - - Paläolithische und geologische Chronologie. - Z. deutsch. geol. Ges. 91, 57-64.

Penck, A., \& BRÜCKNeR, E. (1901-1909): Die Alpen im Eiszeitalter. Leipzig.

Reich, Helga (1953): Die Vegetationsentwicklung der Interglaziale von Großweil-Ohlstadt und Pfefferbichl im Bayerischen Alpenvorland. - Flora 140, 386-443.

SchönhaLs, E. (1956): Diskussionsbeitrag zum Vortrag von J. FinK. - Actes IV Congrès Internat. du Quaternaire Rome-Pise 1953 S. 322.

Schuster, J. (1909): Paläobotanische Notizen aus Bayern. - Ber. bayer. botan. Ges. 12, 57-62.

Sнotton, F. W. (1959): Insects in Pleistocene deposits. - Journ. Institute of Biology 6, 3.

Shotton, F. W. \& Strachan, I. (1958): Proceed. Geol. Soc. London no. 1557, 34. - (1959): The investigation of a peat moor at Rodbaston, Penkridge, Staffordshire. - Quarterly J. geol. Soc. London 115, 1-16.

Simpson, I. M., \& WEST, R. G. (1958): On the stratigraphy of a late-Pleistocene organic deposit at Chelford, Cheshire. - New Phytol. 57, 239.

VÉRTes, L. (1959): Untersuchungen an Höhlensedimenten. - Régészeti Füzetek Ser. II, 7, Beiblatt, $1-176$, Budapest.

VRIES, Hl. de (1959a): Radiocarbon dating of the fossil soils at Ober Fellabrunn. - Proceed. Akad. Wetensch. Amsterdam, Ser. B, 62, No. 1, Physics, 84-91. - - (1959b): in F. Felgenhauer, J. FinK, Hl, de VRIES 1959, 69-73.

WoldstedT, P. (1958a): Eine neue Kurve der Würm-Eiszeit. - EuG 9, 151-154. - - (1958b): Das Eiszeitalter. 2. Aufl. 2. Bd. Stuttgart.

Zeuner, F. E. (1954): Riß or Würm? - EuG 4/5, 98-105.

Anschrift des Verf.: Dr. Hugo Groß, Bamberg, Kunigundendamm 59. 